\title{
Effect of activity level after a posterior-stabilized TKA on the relative bone mineral density measured on standard radiographs in periprosthetic tibial bone
}

\section{Yong Liu}

Sun Yat-sen University First Affiliated Hospital

\section{Peiheng He}

Sun Yat-sen University First Affiliated Hospital

\section{Xing Li}

Sun Yat-sen University First Affiliated Hospital

\section{Yongheng Ye}

Sun Yat-sen University First Affiliated Hospital

\section{Minghao Liu}

Sun Yat-sen University First Affiliated Hospital

\section{Weizhi Chen}

Sun Yat-sen University First Affiliated Hospital

Dongliang XU ( $\square$ dddrxu@163.com )

Sun Yat-Sen University https://orcid.org/0000-0002-0927-5778

Research article

Keywords: TKA, BMD, UCLA, Activity level

Posted Date: December 31st, 2020

DOl: https://doi.org/10.21203/rs.3.rs-37186/v4

License: (c) (1) This work is licensed under a Creative Commons Attribution 4.0 International License. Read Full License 


\section{Abstract}

Background: The aim of this study was to evaluate the effect of activity level after a posterior-stabilized total knee arthroplasty (TKA) on the relative bone mineral density (rBMD) measured on standard radiographs in periprosthetic tibial bone.

Methods: A retrospective review identified 110 patients (110 knees $₫ 20$ men/90 women) who underwent PS TKA with 5 years follow-up. Patients activity level was evaluated by University of California Los Angeles (UCLA) activity score, and the rBMD in periprosthetic tibial bone was measured on anteroposterior X-ray images. Clinical assessments included Western Ontario and McMaster Universities (WOMAC), Knee Society score (KSS) and visual analogue scale (VAS). Nonlinear regression analysis was used to assess the impact of activity levels on periprosthesis bone density.

Results: During 5-year follow-up period, the bone density in the medial, lateral and distal areas decreased compared with that before surgery $(p<0.0001)$. There was a U-shaped distribution between UCLA activity rating and rBMD loss, with the lowest rBMD loss when the UCLA activity score was between 6-8 at 1 and 3 years. The curve fitting of UCLA activity level and rBMD\% showed there was a parabolic relationship between UCLA activity level and rBMD\% at 1 and 3 years after surgery $(P<0.001, P=0.001)$, while there was no significant relationship between UCLA activity level and rBMD\% at 5 years after surgery $(P=0.436)$.

Conclusions: We found that physical activity had a significant effect on radiographic measurements of BMD at 1 and 3 years but not at 5 years. Moderate activity may be associated with less proximal tibial BMD loss after TKA, therefore it may be the most appropriate activity intensity for patients with TKA.

\section{Background}

An increasing number of patients with end-stage osteoarthritis are receiving treatment with total knee arthroplasty (TKA), which is a successful procedure for alleviating pain, improving functional ability, and enhancing quality of life[1]. Bone mineral density (BMD) is an indicator of bone quality and reflects the material properties, bone metabolism and risk of fracture[2, 3]. Postoperative changes in the periprosthetic bone density in the tibia are closely related to the outcomes of TKA[4, 5]. Several studies[69] have reported a reduction in BMD in the proximal tibia after TKA, which can cause the subsidence of some components, especially of the tibial plateau, and increase the risk of prosthetic loosening and further revision.

Physical activity is a critical part of functional recovery of the knee joint after TKA[10]. In clinical practice, it is found that the gray value ( $X$ rays) of patients who actively engage in functional exercises is significantly brighter than that of patients who stay in bed for a long time or not take functional exercises, suggesting that the physical activity is related to the BMD around the prosthesis. Oktas et al[11]concluded that a rehabilitation program for strengthening the muscles around the hip and knee joints was quite important to the success of TKA. However, Kilgus, DJ et al[12] reported that a high level 
of physical activity was correlated with increased osteoporosis-related prosthetic loosening. Therefore, we hypothesized that the physical activity after TKA would influence the bone density around the prosthesis. The aim of this study was to evaluate the effect of activity level after a posterior-stabilized TKA on the relative bone mineral density measured on standard radiographs in periprosthetic tibial bone.

\section{Methods}

\section{Study design}

This was a retrospective study based on data collected from consecutive patients undergoing primary TKA from January 2010 to December 2015. Postoperative assessments were conducted at 1 week, 3 months, 1 year, 3 years, and 5 years after surgery. This study was approved by the Medical Ethics Committee of The First Affiliated Hospital of Sun Yat-Sen University (code number [2011] 57), and all the procedures followed the principles of the Helsinki Declaration.

\section{Patients enrollment}

A total of 121 patients with a diagnosis of knee osteoarthritis (Kellgren-Lawrence classification III or IV) were enrolled in the research, 11 patients were excluded because of incomplete data. At last, 110 patient data were included in the study and analyzed. The mean age of the patients ( 90 females, 20 males) was 66.6 \pm 7.4. Patients were considered eligible if they met the following criteria: More than 50 years old; Reported the presence of knee pain greater than 6 months; diagnosed with knee arthritis by a surgeon; treated surgically with a KTA. Patients were excluded based on the following: varus deformity greater than $15^{\circ}$ هrheumatoid arthritis as the primary indication for surgery; drugs that affect bone mineral density were used; Body Mass Index (BMI) was greater than 30; Failure to complete long-term follow-up.

The patients accepted unilateral primary cemented TKA by the same experienced surgeon approximately 1 week after admission in the Department of Joint Surgery of the First Affiliated Hospital of Sun Yat-Sen University. A posterior-stabilized prosthesis (DePuy Synthes, P.F.C. Sigma, Warsaw, IN, USA) was implanted for TKA.

\section{rBMD (calibrated grayscale value) measurements}

Dual X-ray absorptiometry (DXA) is the golden standard used to evaluate BMD, but it is not used in routine examinations after TKA. Hernandez-Vaquero et al[13] reported a method based on digital X-rays images to evaluate BMD, and the consistency between the BMD measured by DXA and the relative BMD (rBMD ) measured by this method was approximately 0.72 to 0.92 . Therefore, this method was used to evaluate BMD in this study.

In order to ensure the comparability between the $\mathrm{X}$ images, all $\mathrm{X}$-rays were taken on the same machine, and the patient's knee joint was controlled in a neutral position, so as to reduce the errors caused by the 
difference in the position of knee. Knee flexion was minimized by fixing the tibial tubercle at the lower end of the knee. Rotation was controlled by fixing the heel and the first and second toes. Ten regions of interest (ROIs) were chosen as the measured regions in tibia: four lateral regions $(L 1, L 2, L 3, L 4)$, four medial regions (M1, M2, M3, M4), and two distal regions (D1, D2) (Fig 1). ImageJ, (version1.8, NIH, USA) was used to measure the mean grayscale value in the established regions of the radiographs. The measured grayscale value of each designated region was calibrated using the formula:, where $G_{C, R}$ is the calibrated grayscale value, also representing the rBMD in a given region, $G_{R}$ is the grayscale value within an ROI, $G_{a}$ is the value of air within the radiograph, and $G_{f}$ is the grayscale value of the femoral component.

The medial tibial rBMD was defined as the mean values of $M 1, M 2$ and $M 3$, the lateral tibial rBMD as the mean values of $L 1, L 2$ and $L 3$, and the distal tibial plateau as the mean values of D1 and D2, and the tibial rBMD as the mean values of 10 ROIs.

\section{Clinical data collection}

Data of gender, age, BMI, Operative Duration, Kellgren-Lawrence Classification, length of Stay were obtained through patient medical records. The hip-knee-ankle (HKA) angle was measured by X-ray. All patients were clinically evaluated with respect to knee function using the Knee Society Score (KSS)[14], the Western Ontario and McMaster University Osteoarthritis Index (WOMAC)[15] and visual analogue scale (VAS) score. The level of activity was evaluated using the University of California Los Angeles (UCLA) Activity Rating Scale[16, 17].

\section{Statistical analysis}

All statistical analyses were performed using SPSS, Version 21.0. (SPSS Inc., Chicago, IL, USA). The Shapiro-Wilk test was used to confirm that the data were normally distributed. One-way ANOVA was used to compare clinical scores and rBMD at different time points. To verify the influence of other factors on rBMD\%, Pearson's chi-squared test was used to examine the association between sex, age, HKA angle, $\mathrm{BMI}$ and $\mathrm{rBMD} \%$. Curve fitting and nonlinear regression were performed to clarify the relationship between the UCLA activity rating and $\mathrm{rBMD} \%$ at different time point. Post hoc power analyses were performed after the study. Significance was defined as $P<0.05$.

\section{Results}

At last, a total of 110 patients ( 20 men/90women) were included in the analysis. Post hoc power analysis suggested that our sample size was sufficient. The mean age was 66 years. Details of the patients are 
shown in Table 1. A proportion of patients (9\%) were excluded from the analysis due to lack of complete data. There no significant differences between the characteristics of the included and excluded patients.

\section{Clinical outcome-WOMAC, KSS and VAS}

By the 5-year follow-up examination, the knee score and function score (WOMAC, KSS, VAS score) for each study participant had improved significantly over preoperative scores $(P<0.001$, Table 2$)$.

\section{Changes in tibial rBMD after TKA}

During 5-year follow-up period, the bone density in the medial, lateral and distal areas decreased compared with that before surgery $(p<0.0001)$. As shown in Figure 2 and Table 3 , compared with the baseline, rBMD of medial side, lateral side and distal side decreased by $3.0 \%, 4.2 \%$ and $1.4 \%$ respectively at 3 months, decreased by $3.5 \%, 5.1 \%$ and $1.4 \%$ respectively at 1 year, decreased by $4.0 \%$, $5.6 \%$ and $1.8 \%$ respectively at 3 years, and decreased by $6.7 \%, 9.7 \%$ and $3.3 \%$ respectively at 5 years.

\section{Effect of activity level after TKA on rBMD in tibial}

Pearson's chi-squared test showed that there were no significant association between them (Table 4). To verify the effect of postoperative activity level (UCLA activity scale) on rBMD\%, a line graph of UCLA activity scale and $\mathrm{rBMD} \%$ was drawn and the results showed that there was a U-shaped distribution between them $\varangle$ with the lowest rBMD loss when the UCLA activity score was between 6-8 at 1 and 3 years (Fig 3, Table 5).

To find relation between UCLA activity rating and rBMD\%, the curve fitting has been employed (Fig 4). The curve fitting of UCLA activity rating and $\mathrm{rBMD} \%$ showed there was a parabolic relationship between UCLA activity rating and $r B M D \%$ at 1 and 3 years after surgery $(P<0.001, P=0.001)$, while there was no significant relationship between UCLA activity rating and rBMD\% at 5 years after surgery $(P=0.436)$.

\section{Discussion}

This retrospective study revealed that the bone density in periprosthetic tibial decreased after TKA, with the largest decrease at 1 year after surgery, and gradually stabilized in the future; Physical Activity was an important factor that affects bone mineral density; The effect of physical activity on bone density in periprosthetic tibial was time-dependent.

This research demonstrated a decrease in BMD in the proximal tibia after TKA. The decline was most pronounced in the first year. Li and Nilsson[18] reported that bone density temporarily dropped by 13 percent in the first three months after TKA. Hvid et al. [19]found an $11 \%$ decrease in BMD at two years, whereas Petersen et al. [9]reported a 22\% decrease in BMD after three years of TKA. In clinical practice, bone resorption and osteoporosis were observed around the prosthesis after TKA, and osteoporosis was obviously observed during revision surgery. 
In fact, the decrease in BMD after TKA had been reported and was generally believed to be the result of a biomechanical response to stress shielding of the tibial component[20-23]. TKA surgery altered the intraarticular environment and osteotomies exposed cancellous bone directly to the environment, making the distal tibia more susceptible to environmental change. Prosthesis implantation produced stress shielding effect on bone, so the stress load on bone was reduced, which leaded to increased bone absorption, decreased bone mass, and decreased bone density. On the other hand冈long-term interaction between the prosthesis and bone resulted in wear particles, which in turn leaded to aseptic inflammation and aggravated osteolysis.

It was found that the activity level had a bidirectional effect on the bone density around the prosthesis. A nonlinear (parabolic) relationship between the UCLA activity level and rBMD\% in the proximal tibia suggested that moderate physical activity was beneficial to maintain bone mass, while insufficient or excessive functional exercise aggravated bone loss. This phenomenon is consistent with Wolff's law[24], which indicates that mechanical stress stimulates bone formation, while disuse leads to bone loss. Petersen et al[9] revealed that a decreased load led to rapid bone loss, while an increased load led to a small increase in BMD in the tibial condyles. Gallo, $\mathrm{J}$ et al. [25] reported that for man, high levels of physical activity, and a high historical level of physical activity were associated with a higher risk of reoperation; for women, the early reoperation was associated with lower physical activity.

The effect of activity level on bone mineral density was correlated with time. The results showed that the activity level had a significant effect on bone density at 1 year and 3 years after surgery, but no significant at 5 years. The significant influence of activity level on rBMD at 1 and 3 years could be attributed to adaptive bone remodeling and stress shielding effect, as Jaroma, A et al. reported that tibial metaphyseal periprosthetic bone was remodeled after TKA due to mechanical axis correction [8]. Seitz et al[26] found that there was a reparation phase and a stabilization phase during bone remodeling period after TKA and no significant BMD change was observed during the stabilization phase. After long-term interaction between bone and prosthesis, a stable state was formed, which was manifested as a close bond between bone and prosthesis and a fusion of bone and prosthesis interface. In such a stable state, bone mineral density was less or even not affected by external environment. This study showed that 5 years after TKA, the bone mineral density in proximal tibial tended to be stable, and activity level had no significant effect on bone mineral density.

Our results revealed 3 important findings $\varangle$ First, the bone density in periprosthetic tibial decreased after TKA; Second, physical activity was an important factor affecting bone density in periprosthetic tibial; Third, the influence of physical activity on bone density was bidirectional and time-dependent. Clinically, prosthesis instability, periprosthetic fractures, and proximal tibial osteoporosis are closely related to periprosthetic bone density decrease after TKA. Therefore, according to the changes of bone density after TKA, appropriate intervention therapy should be conducted to guide postoperative rehabilitation, so as to reduce bone loss in proximal tibial and improve clinical efficacy. Firstly, in view of the decrease of bone density in periprosthetic tibial after TKA, interventions that can improve bone mineral density should be taken in postoperative rehabilitation period, such as body weight control, oral drugs to improve bone 
mineral density, and proper functional exercises. Secondly, because activity level had an impact on bone mineral density, and appropriate activity level could reduce bone density loss, moderate level of functional exercise should be conducted after TKA. Thirdly, the effect of activities on bone mineral density was time-dependent, so patients should have appropriate functional exercise activities in the early postoperative period.

There are some limitations in our study. First $\$ this was a retrospective study, with selective bias and confounders bias, and the number of participants in the study was limited. Second, no control group was established in this study. Third, the sex distribution was atypical with $82 \%$ females. However, according to relevant statistics, the ratio of male to female knee osteoarthritis is about 1:7, so female subjects are the majority. Forth, the BMD measured on standard X-ray images is a relative value. Although the rBMD measured on standard X-ray images is not the true BMD, Hernandez-Vaquero et al[13] have proven that the relationship between the rBMD measured on standard X-ray images and the true BMD measured by DXA is linear, therefore, rBMD could serve as an alternative to BMD.

\section{Conclusion}

In this retrospective study, we found that the bone density in periprosthetic tibial decreased after TKA and physical activity had a significant effect on radiographic measurements of BMD at 1 and 3 years but not at 5 years. Moderate activity may be associated with less proximal tibial BMD loss after TKA, therefore it may be the most appropriate activity intensity for patients with TKA.

\section{List Of Abbreviations}

TKA: total knee arthroplasty, BMD: bone mineral density, rBMD: relative BMD, rBMD\%: the percent change in rBMD, DXA: Dual X-ray absorptiometry, ROls: regions of interest, KSS: Knee Society Score, WOMAC: Western Ontario and McMaster University Osteoarthritis Index, VAS: visual analogue scale, UCLA activity rating : University of California Los Angeles Activity Rating Scale, BMl: Body Mass Index , HKA angle: hipknee-ankle angle, ANOVA: Analysis of Variance.

\section{Declarations}

- Ethics approval and consent to participate

This study was approved by the Medical Ethics Committee of The First Affiliated Hospital of Sun Yat-Sen University (code number [2011] 57), and all the procedures followed the principles of the Helsinki Declaration.

- Consent for publication

Not applicable 
- Availability of data and materials

The datasets used and/or analysed during the current study are available from the corresponding author on reasonable request.

- Competing interests

The authors declare that they have no competing interests

- Funding

This work was supported by the 5010 Clinical Research Project of Sun Yat-sen University (2010005).

- Authors' contributions

YL and DX conceived the project, YL designed the experiment, collected and analyzed patients' data, and wrote the manuscript, PH participated in patient follow-up and provided comments, YY, XL, ML and WC assisted in patient follow-up. All authors read and approved the final manuscript.

- Acknowledgements

Not applicable

\section{References}

1. Charlesworth J, Fitzpatrick J, Perera NKP, Orchard J: Osteoarthritis- a systematic review of long-term safety implications for osteoarthritis of the knee. BMC Musculoskelet Disord 2019, 20(1):151.

2. Small SR, Ritter MA, Merchun JG, Davis KE, Rogge RD: Changes in tibial bone density measured from standard radiographs in cemented and uncemented total knee replacements after ten years' followup. Bone \& Joint Surgery 2013, 95-B:911-916.

3. Sessa G, Costarella L, Puma Pagliarello C, Di Stefano A, Sessa A, Testa G, Pavone V: Bone mineral density as a marker of hip implant longevity: a prospective assessment of a cementless stem with dual-energy X-ray absorptiometry at twenty years. International orthopaedics 2019, 43(1):71-75.

4. Li Y, Ou Y, Zhu Y, Zhao Z, He B, Xu S, Yu H: The predictive significance of bone mineral density on postoperative pain relief in knee osteoarthritis patients after total knee arthroplasty: A prediction model. J Orthop Sci 2020.

5. Bernatz JT, Krueger DC, Squire MW, Illgen RL, 2nd, Binkley NC, Anderson PA: Unrecognized Osteoporosis Is Common in Patients With a Well-Functioning Total Knee Arthroplasty. The Journal of arthroplasty 2019, 34(10):2347-2350.

6. Ritter MA, Davis KE, Small SR, Merchun JG, Farris A: Trabecular bone density of the proximal tibia as it relates to failure of a total knee replacement. Bone Joint J 2014, 96b(11):1503-1509. 
7. Winther $\mathrm{N}$, Jensen $\mathrm{C}$, Petersen $\mathrm{M}$, Lind $\mathrm{T}$, Schroder $\mathrm{H}$, Petersen $\mathrm{M}$ : Changes in bone mineral density of the proximal tibia after uncemented total knee arthroplasty. A prospective randomized study. International orthopaedics 2016, 40(2):285-294.

8. Jaroma A, Soininvaara T, Kroger H: Periprosthetic tibial bone mineral density changes after total knee arthroplasty. Acta orthopaedica 2016, 87(3):268-273.

9. Petersen MM, Nielsen PT, Lauritzen JB, Lund B: Changes in bone mineral density of the proximal tibia after uncemented total knee arthroplasty. A 3-year follow-up of 25 knees. Acta Orthop Scand 1995, 66(6):513-516.

10. Larsen JB, Mogensen L, Arendt-Nielsen L, Madeleine P: Intensive, personalized multimodal rehabilitation in patients with primary or revision total knee arthroplasty: a retrospective cohort study. BMC Sports Sci Med Rehabil 2020, 12:5.

11. Oktas $B$, Vergili $O$ : The effect of intensive exercise program and kinesiotaping following total knee arthroplasty on functional recovery of patients. J Orthop Surg Res 2018, 13(1):233.

12. Kilgus DJ, Dorey FJ, Finerman GA, Amstutz HC: Patient activity, sports participation, and impact loading on the durability of cemented total hip replacements. Clin Orthop Relat Res 1991(269):25-31.

13. Hernandez-Vaquero D, Garcia-Sandoval MA, Fernandez-Carreira JM, Suarez-Vazquez A, PerezHernandez D: Measurement of bone mineral density is possible with standard radiographs - A study involving total knee replacement. Acta orthopaedica 2005, 76(6):791-795.

14. Giesinger JM, Hamilton DF, Jost B, Behrend H, Giesinger K: WOMAC, EQ-5D and Knee Society Score Thresholds for Treatment Success After Total Knee Arthroplasty. The Journal of arthroplasty 2015, 30(12):2154-2158.

15. Walker LC, Clement ND, Deehan DJ: Predicting the Outcome of Total Knee Arthroplasty Using the WOMAC Score: A Review of the Literature. J Knee Surg 2019, 32(8):736-741.

16. Low AHL, Xin X, Law WG, Teng GG, Santosa A, Lim A, Chan G, Ng SC, Thumboo J: Validation of the UCLA Scleroderma Clinical Trial Consortium Gastrointestinal Tract Instrument 2.0 in English- and Chinese-speaking patients in a multi-ethnic Singapore systemic sclerosis cohort. Clin Rheumatol 2017, 36(7):1643-1648.

17. Zahiri CA, Schmalzried TP, Szuszczewicz ES, Amstutz HC: Assessing activity in joint replacement patients. The Journal of arthroplasty 1998, 13(8):890-895.

18. Li MG, Nilsson KG: Changes in bone mineral density at the proximal tibia after total knee arthroplasty: A 2-year follow-up of $\mathbf{2 8}$ knees using dual energy x-ray absorptiometry. J Orthopaed Res 2000, 18(1):40-47.

19. Hvid I, Bentzen SM, Jorgensen J: Remodeling of the tibial plateau after knee replacement. CT bone densitometry. Acta Orthop Scand 1988, 59(5):567-573.

20. Lonner JH, Klotz M, Levitz C, Lotke PA: Changes in bone density after cemented total knee arthroplasty: influence of stem design. The Journal of arthroplasty 2001, 16(1):107-111.

21. Soininvaara TA, Miettinen HJA, Jurvelin JS, Suomalainen OT, Alhava EM, Kröger HPJ: Periprosthetic tibial bone mineral density changes after total knee arthroplastyखone-year follow-up study of 69 
patients. Acta Orthop Scand 2004, 75 (5):5.

22. Abu-Rajab RB, Watson WS, Walker B, Roberts J, Gallacher SJ, Meek RM: Peri-prosthetic bone mineral density after total knee arthroplasty. Cemented versus cementless fixation. $J$ Bone Joint Surg $\mathrm{Br}$ 2006, 88(5):606-613.

23. Prince JM, Bernatz JT, Binkley N, Abdel MP, Anderson PA: Changes in femoral bone mineral density after total knee arthroplasty: a systematic review and meta-analysis. Arch Osteoporos 2019, 14(1):11.

24. Enlow DH: Wolff's law and the factor of architectonic circumstance. Am J Orthod 1968, 54(11):803822.

25. Gallo J, Kriegova E, Kudelka M, Lostak J, Radvansky M: Gender Differences in Contribution of Smoking, Low Physical Activity, and High BMI to Increased Risk of Early Reoperation After TKA. The Journal of arthroplasty 2020, 35(6):1545-1557.

26. P S, P R: CT bone densitometry of the anchorage of artificial knee joints. J Comput Assit Tomogr 1985(9):607-608.

\section{Tables}

Table 1 Patient characteristics

\begin{tabular}{|ll|}
\hline Characteristic* $^{*}$ & Mean (SD, range) \\
\hline Age & $66.6(7.4,50-87)$ \\
\hline Sex $(\mathrm{n})$ & 20 men/90women \\
BMI $\left(\mathrm{kg} / \mathrm{m}^{2}\right)$ & $26.6(3.4,18.6-39.5)$ \\
\hline HKA angle & $180.4(1.5,175-185)$ \\
\hline Operative duration $(\mathrm{min})$ & $135.0(36.2,60-270)$ \\
\hline LOS (day) & $11.6(2.9,7-22)$ \\
\hline Kellgren-Lawrence classification & \\
\hline III & 40 \\
\hline IV & 70 \\
\hline
\end{tabular}

* BMI, body mass index; HKA, hip-knee-ankle, after surgery; LOS, length of stay.

Table 2 Clinical evaluation of participants $(\mathrm{N}=110)$ 


\begin{tabular}{|lllllll|}
\hline & Preoperative & 3 months & 1 year & 3 years & 5 years & $P$ \\
\hline KSS score & $77.9 \pm 24.3$ & $120.3 \pm 13.4$ & $165.0 \pm 8.6$ & $170.4 \pm 8.9$ & $182.1 \pm 8.8$ & $<0.001$ \\
\hline WOMAC score & $43.9 \pm 13.2$ & $27.1 \pm 11.5$ & $10.1 \pm 3.4$ & $5.5 \pm 2.9$ & $3.4 \pm 1.9$ & $<0.001$ \\
\hline VAS score & $6.8 \pm 1.2$ & $5.63 \pm 1.3$ & $2.3 \pm 1.3$ & $1.4 \pm 0.4$ & $1.2 \pm 0.7$ & $<0.001$ \\
\hline
\end{tabular}

Note: Values are the mean \pm SD. $P$ value is compared between 5 years after surgery and preoperative by $t$ test.

Table 3 rBMD in Lateral, Medial and Distal region at different time point

\begin{tabular}{|lllllll|}
\hline & baseline & 3months & 1year & 3years & 5 years & $P$ \\
\hline $\mathrm{N}$ & 110 & 110 & 110 & 110 & 110 & - \\
\hline $\mathrm{L}$ & $120.2 \pm 6.8$ & $115.1 \pm 8.3$ & $114.1 \pm 9.4$ & $113.5 \pm 9.9$ & $108.6 \pm 9.1$ & $<0.0001$ \\
\hline $\mathrm{M}$ & $116.7 \pm 8.2$ & $113.2 \pm 8.4$ & $112.6 \pm 9.6$ & $112.0 \pm 10.9$ & $108.9 \pm 9.4$ & $<0.0001$ \\
\hline $\mathrm{D}$ & $133.9 \pm 8.2$ & $132.0 \pm 7.9$ & $132.0 \pm 7.8$ & $131.5 \pm 7.6$ & $129.5 \pm 7.2$ & 0.72 \\
\hline
\end{tabular}

Note: $\mathrm{N}$ is the number of participants; $\mathrm{L}$ is lateral region; $\mathrm{M}$ is medial region; $\mathrm{D}$ is distal region.

Table 4 Pearson's chi-squared test for sex, age, hip-knee-ankle (HKA) angle, BMI and rBMD\%

\begin{tabular}{|lllllll|}
\hline & rBMD\% & & & & & \\
& 1 year & & & & \\
& $\begin{array}{l}\text { Pearson } \\
\text { correlation }\end{array}$ & $P$ & $\begin{array}{l}\text { Pearson } \\
\text { correlation }\end{array}$ & $P$ & $\begin{array}{l}\text { Pearson } \\
\text { correlation }\end{array}$ & $P$ \\
\hline Sex & 0.069 & 0.473 & 0.100 & 0.297 & 0.065 & 0.50 \\
\hline Age & 0.159 & 0.097 & 0.045 & 0.639 & 0.001 & 0.995 \\
\hline $\begin{array}{l}\text { HKA } \\
\text { angle }\end{array}$ & 0.052 & 0.592 & 0.059 & 0.541 & 0.094 & 0.330 \\
\hline BMl & 0.118 & 0.219 & 0.010 & 0.918 & 0.018 & 0.848 \\
\hline
\end{tabular}


Note: Pearson's chi-squared test was used to examine the association between sex, age, hip-knee-ankle (HKA) angle, BMI and rBMD\%, the results showed that there were no significant association between them.

Table 5 rBMD\% at different activity levels and different time point

\begin{tabular}{|lllllll|}
\hline UCLA & \multicolumn{2}{l}{ rBMD\% } & & & & \\
activity level & $1 \mathrm{y}$ & $\mathrm{N}$ & $3 \mathrm{y}$ & $\mathrm{N}$ & $5 \mathrm{y}$ & $\mathrm{N}$ \\
\hline 4 & $6.6 \pm 5.2$ & 12 & $8.2 \pm 4.3$ & 14 & $6.6 \pm 1.7$ & 11 \\
\hline 5 & $5.3 \pm 5.3$ & 15 & $5.7 \pm 5.9$ & 13 & $7.4 \pm 4.9$ & 16 \\
\hline 6 & $0.5 \pm 2.6$ & 22 & $0.6 \pm 3.1$ & 26 & $5.8 \pm 3.6$ & 22 \\
\hline 7 & $1.5 \pm 2.9$ & 20 & $-0.2 \pm 3.1$ & 16 & $6.9 \pm 3.9$ & 19 \\
\hline 8 & $0.8 \pm 3.5$ & 18 & $2.1 \pm 4.9$ & 19 & $4.8 \pm 4.8$ & 20 \\
\hline 9 & $6.1 \pm 4.2$ & 23 & $7.3 \pm 4.3$ & 22 & $7.2 \pm 4.0$ & 22 \\
\hline
\end{tabular}

Note: Values are the mean \pm SD. $N$ is the number of participants; $r B M D \%=100 \% *[r B M D$ (baseline) $r$ rBMD (each time)]/ rBMD (baseline). A positive value indicates a decrease in rBMD, while a negative value indicates an increase in rBMD.

\section{Figures}




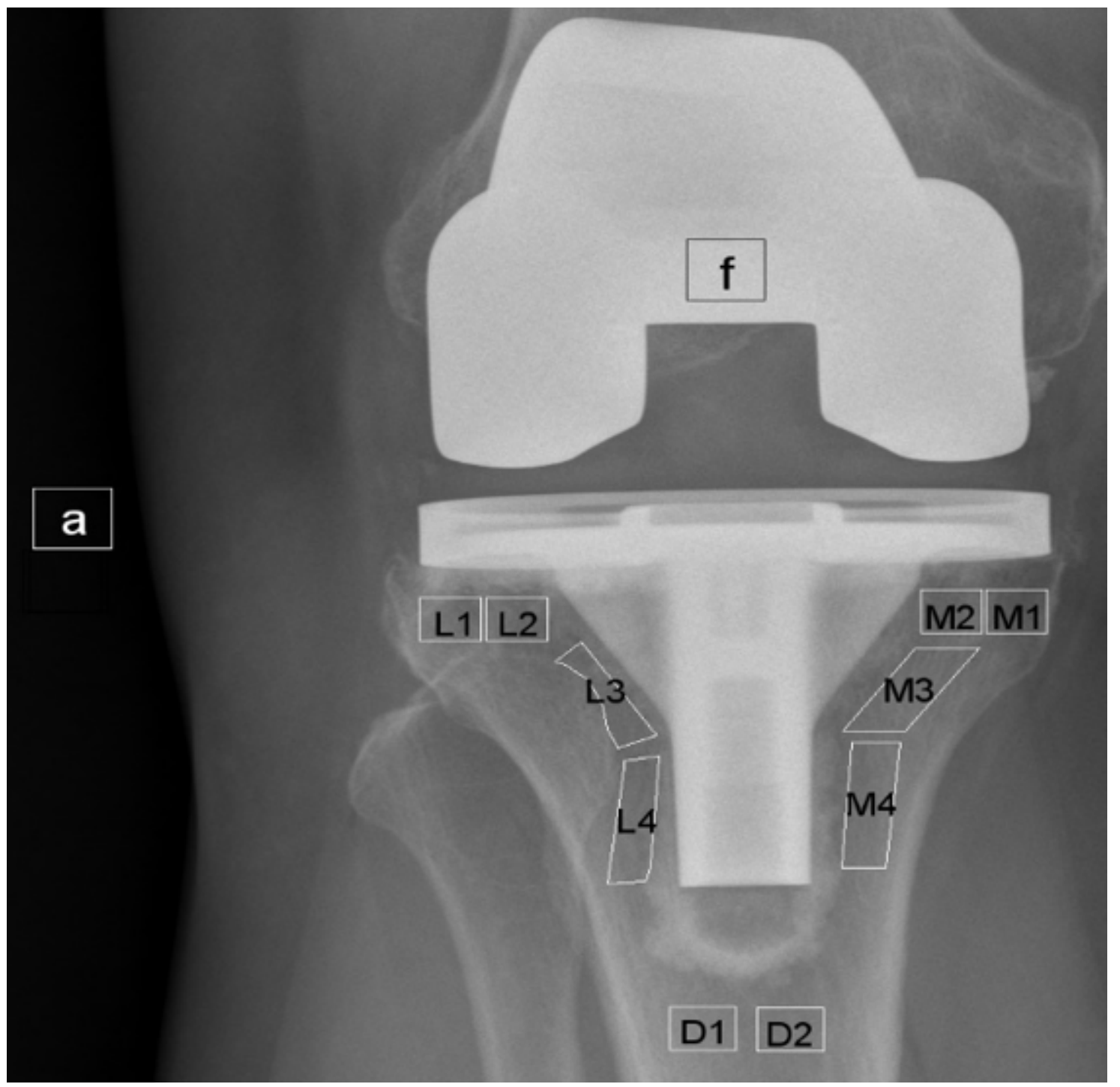

\section{Figure 1}

The rBMD was measured at 10 regions of interest around the tibial component on X-ray film, with 4 medial measurement areas: M1, M2, M3 and M4; 4 lateral measurement areas: L1, L2, L3 and L4; and 2 distal measurement areas: D1 and D2. In the figure above region A and F measure gray values of air and metal of femoral prosthesis respectively to adjust bone density. The measured area avoided cement, cortical bone, and fibula head. The mean value of rBMD around the tibial component was defined as $(M 1+M 2+M 3+M 4+L 1+L 2+L 3+L 4+D 1+D 2) / 10$. The medial mean $r B M D$ was defined as $(M 1+M 2+M 3+M 4) / 4$. The lateral mean $r B M D$ is defined as $(L 1+L 2+L 3+L 4) / 4$. 


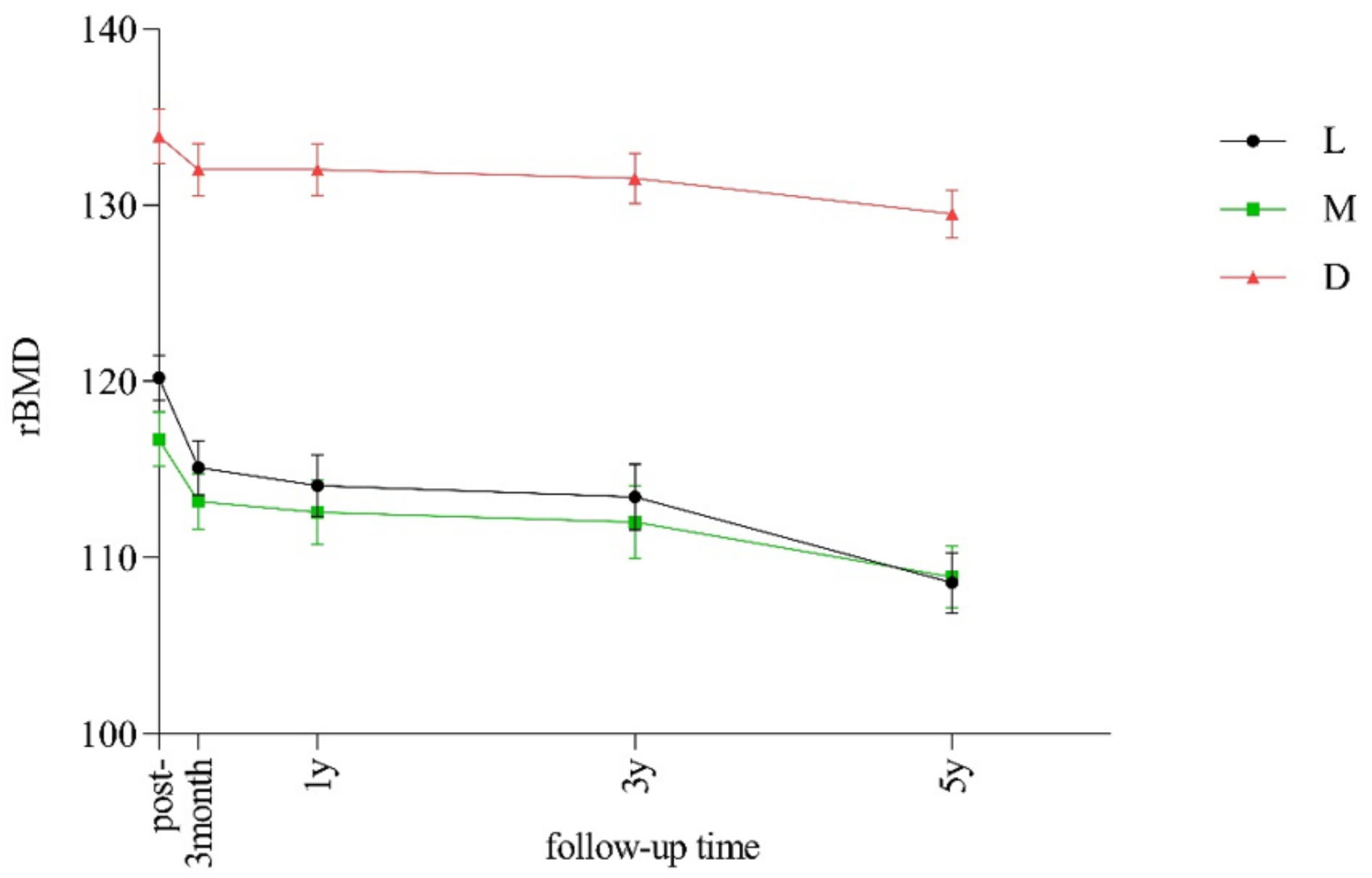

Figure 2

Curves of mean rBMD in medial, lateral and distal regions. rBMD decreased at all time points after surgery compared with baseline. The distal average rBMD of prosthesis was higher than that of medial and lateral average rBMD, while the medial average BMD decreased more than that of lateral average BMD. L囚lateral; M:medial; D:distal. 


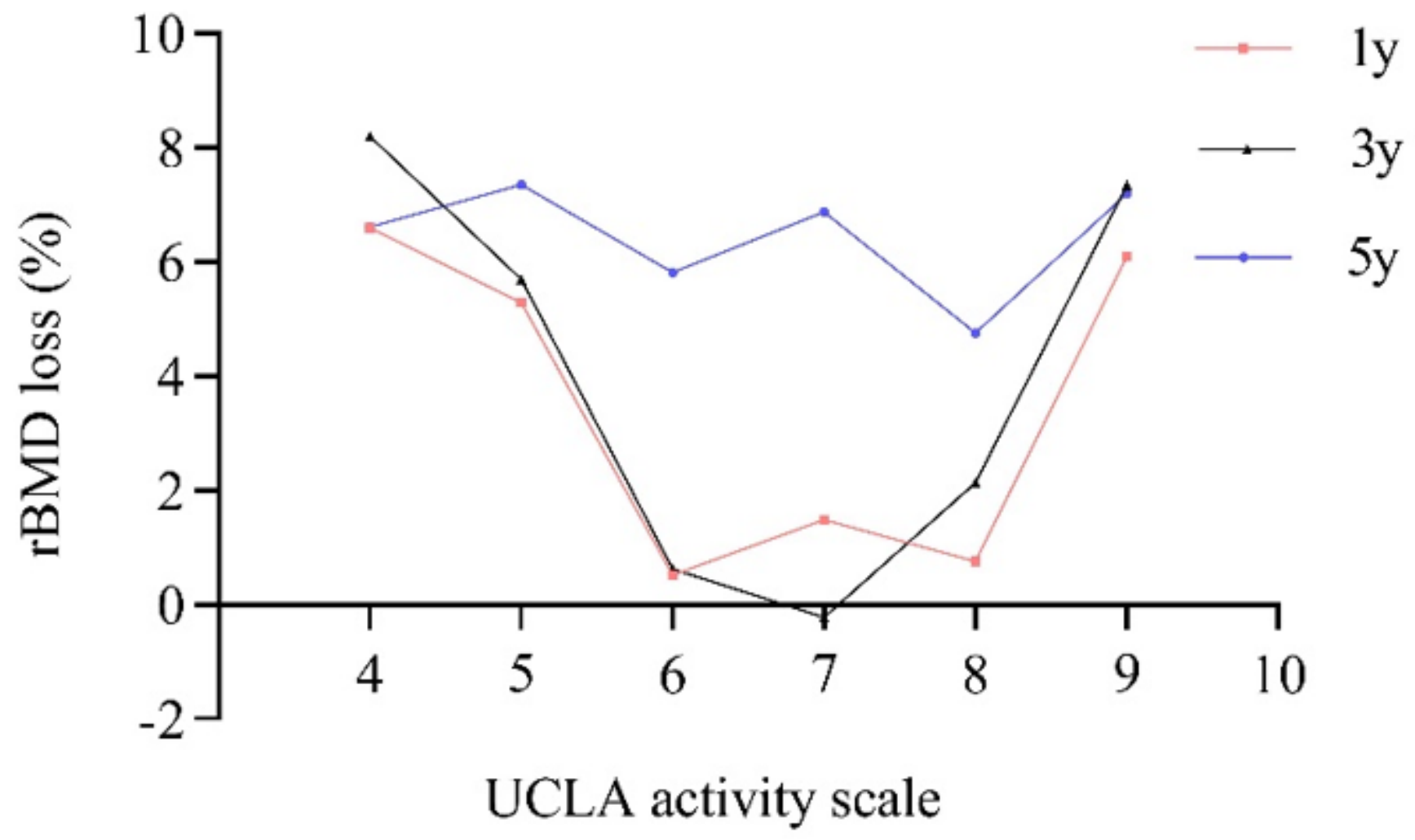

Figure 3

Relationship of UCLA activity rating with rBMD\%. The rBMD measured at 1 week after surgery was defined as baseline. $r B M D \%=(r B M D$ baseline $-r B M D) / r B M D$ baseline $* 100 \%$, indicating the percentage change of $r B M D$ measured after surgery relative to baseline. rBMD \% represents the percentage decrease of rBMD, since the bone density basically showed a decreasing trend after surgery. It can be seen from the figure that when UCLA was $6-8$, the rBMD\% was the smallest. There was a U-shaped distribution for them with the lowest rBMD loss when the UCLA activity score was between 6-8 for 1 and 3 years. There was no such relationship at 5 years. 


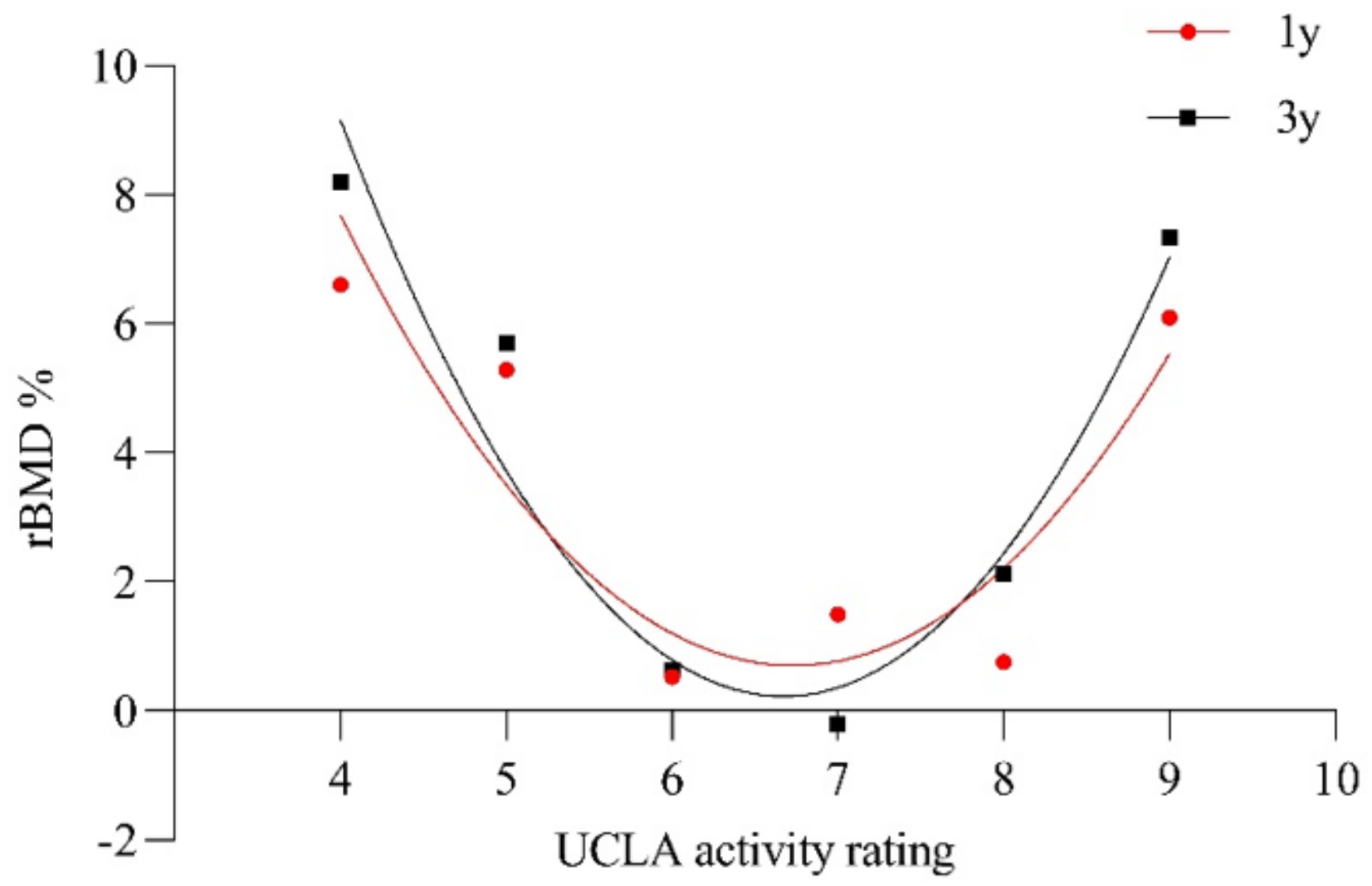

Figure 4

Nonlinear regression model of UCLA activity rating and rBMD\% for measurements at 1 and 3 years. The curve fitting of UCLA activity level and rBMD\% showed there was a parabolic relationship between UCLA activity rating and $r B M D \%$ at 1 and 3 years after surgery $(P<0.001, P=0.001)$, while there was no significant relationship between UCLA activity rating and rBMD\% at 5 years after surgery $(P=0.436)$. This figure showed that postoperative activity level was an important influencing factor of bone density around the prosthesis, and we further discover that the activity level had an effect on the bone around the prosthesis in the early postoperative period, but not in the late postoperative period. Bone density loss around the prosthesis was minimal at activity levels of 6-8. The analysis shows that moderate intensity activity may mitigate against BMD loss around the tibial component. 